\title{
Application of Model Predictive Control on Real-Time Hybrid Simulation
}

\author{
Ping SHAO ${ }^{\mathrm{a}, \mathrm{b}}$, Wei GUO ${ }^{\mathrm{a}, \mathrm{b}}$ and Chen $\mathrm{ZENG}^{\mathrm{a}, \mathrm{b}, 1}$ \\ a School of Civil Engineering, Central South University, Changsha, Hunan Province, \\ 410075, China \\ b National Engineering Laboratory for High Speed Railway Construction, Changsha, \\ Hunan Province, 410075, China
}

\begin{abstract}
Due to the nonlinear behaviour in hydraulic shake table system and control-structure-interaction (CSI) effect, the phase-lag between displacement command and response can be clearly discovered in time domain. This problem is very crucial since the phase-lag of the actuator control can diminish the testing fidelity and might cause numerical instability in real-time hybrid simulation (RTHS). In this paper, the optimal model-based control algorithm named model predictive control (MPC) is adopted to compensate the time delay of the hydraulic shake table control system. The performance of the newly developed MPC is compared to that of the state-of-the-art PID controller numerically. The results show that the proposed MPC algorithm can successfully improve the tracking performance and time delay issue of the hydraulic shake table control system. This fact makes MPC a great method to implement the hydraulic shake table control part for the RTHS application.
\end{abstract}

Keywords. Model predictive control, real-time hybrid simulation, time delay compensation.

\section{Introduction}

For observing and validating the dynamic performance of high-performance structures under different levels of excitations, real-time hybrid simulation (RTHS) could be a promising alternatives or additives to the current shake-table test. In RTHS, it is crucial that the time delay introduced by the hydraulic control system and data acquisition system, is properly compensated to avoid instability and accuracy problems. Horiuchi et al. showed that the delayed response of an actuator adds negative damping to the test structure, resulting in the divergence of the structural response during the testing [1]. Moreover, the installed specimen will interfere the control performance of the shake table, which is so-called control-structure-interaction (CSI) effect [2].

\footnotetext{
${ }^{1}$ Corresponding Author, Chen ZENG, School of Civil Engineering, Central South University, Changsha, Hunan Province, 410075, China; National Engineering Laboratory for High Speed Railway Construction, Changsha, Hunan Province, 410075, China; Email: zengchen2017@csu.edu.cn.
} 
To compensate the time delay issue, researchers have been developing different kinds of compensation techniques. Horiuchi et al. [1] utilized a third order polynomial function to extrapolate and predict the reference displacement command for actuator control. Chae et al. [3] proposed the adaptive time series compensator by updating the coefficients of a second order compensator using a least-square algorithm to minimize the system delay. Recently, the adaptive model reference control strategy is proposed by Amirali and B.F. Spencer [4]. This method combines the adaptive law with the model-based control scheme, which improves the robustness while maintaining the good tracking performance.

In this paper, a model predictive control (MPC) strategy is utilized to compensate the time delay in the real-time shake table control. The dynamic model that contains the hydraulic shake table control system and the installed specimen is modelled, and the parameters are identified by grey-box system identification method for the controller design. Numerical simulation results show that this approach successfully improves the tracking performance and phase response. The robustness against the specimen nonlinearity is also proved in both numerical simulation and real-time experiment.

\section{Description of the Control System and System Identification}

\subsection{Model of Shake Table System with SDOF Specimen}

The control model for the shake table control system considered in this paper is created by physical modeling and parameters identification technique. The hydraulic shake table system including the PID controller, hydraulic actuator, shake table and the tested specimen. The basic schematic is presented in figure 1 .

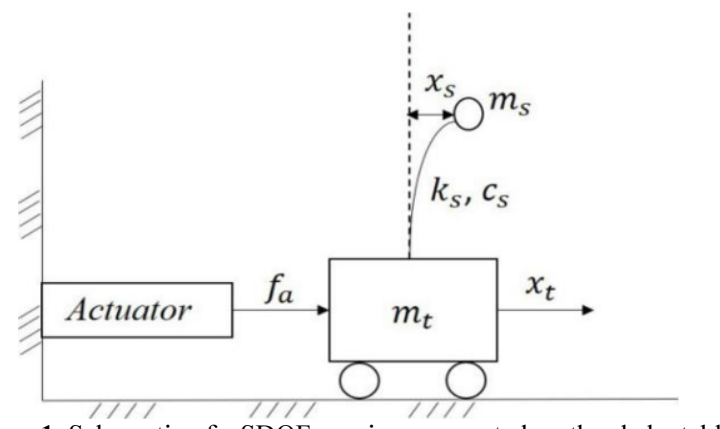

Figure 1. Schematic of a SDOF specimen mounted on the shake table.

Considering the transfer functions of servo valve, actuator and the PID controller, the bare table control system can be represented by a third order system model, which takes displacement command as input and generate displacement feedback as output [5]. The governing equations of the bare table control system expressed in the state space form is given in equation (1), 


$$
\frac{d}{d t}\left[\begin{array}{c}
x_{t} \\
\dot{x}_{t} \\
\frac{f_{a}}{m_{t}}
\end{array}\right]=\left[\begin{array}{ccc}
0 & 1 & 0 \\
0 & 0 & 1 \\
-k_{a} \omega_{a}{ }^{2} & -\omega_{a}{ }^{2} & -2 \xi_{a} \omega_{a}
\end{array}\right]\left[\begin{array}{c}
x_{t} \\
\dot{x}_{t} \\
\frac{f_{a}}{m_{t}}
\end{array}\right]+\left[\begin{array}{c}
0 \\
0 \\
k_{a} \omega_{a}{ }^{2}
\end{array}\right] x_{d}
$$

where, $x_{t}$ and $\dot{x}_{t}$ are the table displacement and velocity; $m_{t}$ is the mass of the shake table; $k_{a}, \omega_{a}$ and $\xi_{a}$ are the control gain, the natural frequency and equivalent damping ratio of the shake table system; $f_{a}$ is the actuator force applied to the shake table; $x_{d}$ is the target displacement.

And the specimen model can be modelled analytically to consider the controlstructure interaction, the governing equations of the specimen can be written as equation (2) and (3).

$$
\begin{aligned}
& m_{s} \ddot{x}_{s}+c_{s} \dot{x}_{s}+k_{s} x_{s}=-m_{s} \ddot{x}_{t} \\
& m_{t} \ddot{x}_{t}-\left(c_{s} \dot{x}_{s}+k_{s} x_{s}\right)=f_{a}
\end{aligned}
$$

where, $x_{s}$ is the relative displacement of the specimen relative to the shake table; $m_{s}$, $k_{s}, c_{s}$ are the mass, stiffness and damping of the specimen, respectively.

Combining equation (1), (2) and (3), the state space form of the shake table system which contains the hydraulic dynamics, and the CSI effect can be given in equation (4),

$$
\frac{d}{d t}\left[\begin{array}{c}
x_{s} \\
\dot{x}_{s} \\
x_{t} \\
\dot{x}_{t} \\
f_{a} \\
m_{t}
\end{array}\right]=\left[\begin{array}{ccccc}
0 & 1 & 0 & 0 & 0 \\
-\left(\frac{1}{m_{s}}+\frac{1}{m_{t}}\right) k_{s} & -\left(\frac{1}{m_{s}}+\frac{1}{m_{t}}\right) c_{s} & 0 & 0 & -1 \\
0 & 0 & 0 & 1 & 0 \\
\frac{k_{s}}{m_{t}} & \frac{c_{s}}{m_{t}} & 0 & 0 & 1 \\
0 & 0 & -k_{a} \omega_{a}^{2} & -\omega_{a}^{2} & -2 \xi_{a} \omega_{a}
\end{array}\right]\left[\begin{array}{c}
x_{s} \\
\dot{x}_{s} \\
x_{t} \\
\dot{x}_{t} \\
\frac{f_{a}}{m_{t}}
\end{array}\right]+\left[\begin{array}{c}
0 \\
0 \\
0 \\
0 \\
k_{a} \omega_{a}^{2}
\end{array}\right] x_{d}
$$

\subsection{Parameters Identification}

The control model for the shake table control system considered in this paper is created by physical modeling approach and parameters identification technique. Several ground motions are used to excite the shake table within the frequency of interest. Both the displacement command and the displacement feedback are recorded to conduct the parameter estimation by grey boxing system identification method. The identified parameters are listed in table 1.

Table 1. Parameters of the shake table control system and SDOF specimen.

$\begin{array}{ll}\text { Mass of the specimen, } m_{s}[\mathrm{~kg}] & 136.5752 \\ \text { Damping coefficient of the specimen, } c_{s} & 1289.667 \\ \text { Stiffness of the specimen, } k_{s}[\mathrm{~N} / \mathrm{mm}] & 297800\end{array}$


Mass of the shake table, $m_{t}$ $575(\mathrm{~kg})$

Control gain of the shake table, $k_{a}$ 60

Damping ratio of the shake table system, $\xi_{a}$

0.8

Natural frequency of the shake table, $\omega_{a}$

232.4779

\section{Model Predictive Control Design}

MPC strategy includes three key processes: prediction, optimization and receding horizon, the basic process is illustrated in figure 2. The general design objective of MPC is to compute a trajectory of a future command to optimize the future response of the shake table.
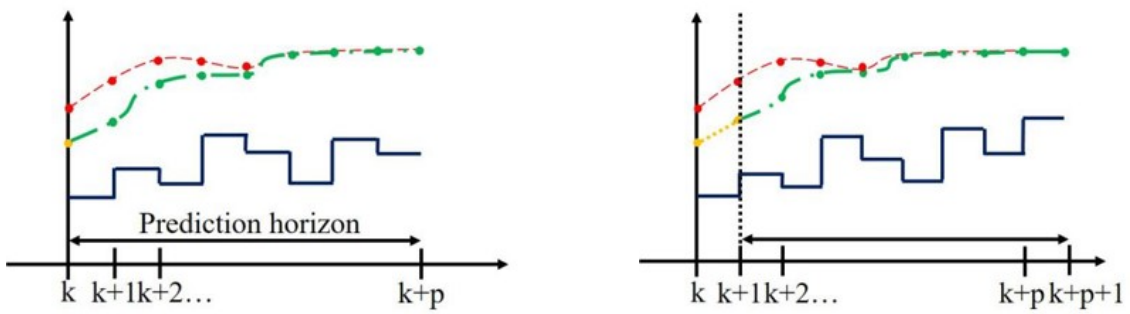

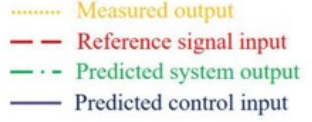

(a) Prediction at time step $k$

Figure 2. Schematic of MPC control strategy.

First, the proposed model in section 2 which is discretized with the sampling frequency equals $1000 \mathrm{~Hz}$, is used in MPC to predict the shake table's response with the future control command as the adjustable variables. Second, MPC will find the optimal control sequence such that a cost function is minimized within the prediction horizon. Third, with the receding horizon control principle, only the first sample in the control sequence is implemented. This procedure is repeated in real time to improve the control performance.

A steady state Kalman filter is designed to obtain the values for the unmeasurable states and predict the future plant output. The filter is designed by solving the discrete algebraic Ricatti equation to get the a priori estimate error covariance $M$ in equation (5). The Kalman gain is designed as constant throughout the entire control process and be calculated in equation (6). With the known Kalman gain, the Kalman filter estimation can be applied, and the steps are presented in equation (7) and (7). By using the states obtained from the steady state Kalman filter in equation (8), the MPC can make predictions for the output variable and solve the optimization problem to generate the optimal control sequence.

$$
\begin{gathered}
A M A^{T}-M+Q-A M C^{T}\left[R+C M C^{T}\right]^{-1} C M A^{T}=0 \\
K=M C^{T}\left(C M C^{T}+R\right)^{-1} \\
x_{c}(k \mid k)=x_{c}(k \mid k-1)+K\left(y_{m}-C x_{c}(k \mid k-1)-D u\right)
\end{gathered}
$$




$$
x_{c}(k+1 \mid k)=A x_{c}(k \mid k)+B u(k)
$$

\section{Numerical Simulation}

\subsection{Numerical Simulation with Linear Specimen}

To investigate the performance of the MPC design strategy, the linear model simulation is conducted by utilizing the parameters identified in table 1 . The control performance of using just PID controller is presented as the benchmark result. The 1980 Irpinia Italy earthquake record is chosen to be the reference signal and the comparison result is presented in figure 3 . It can be clearly shown that both the tracking performance and time delay are improved.

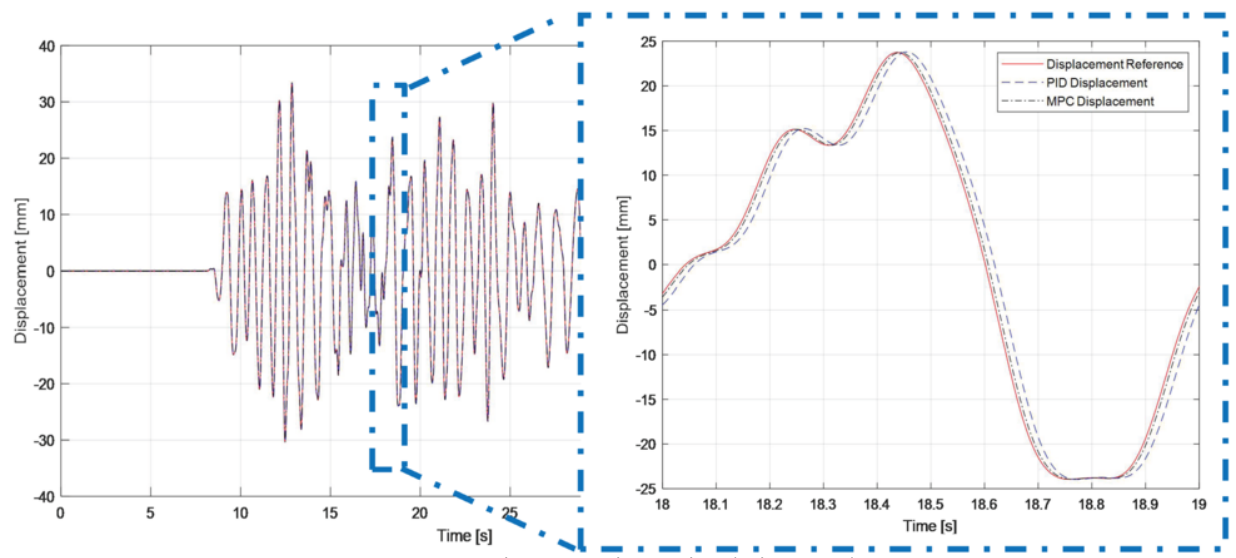

Figure 3. Linear specimen simulation result.

\subsection{Numerical Simulation with Nonlinear Specimen}

To further investigate the effectiveness and robustness of MPC, a nonlinear SDOF specimen is designed in simulation framework to by using the bouc-wen hysteric model [6] presented in equation 5 to represent the specimen nonlinearity and the same MPC controller designed in section 3 is used in the simulation.

$$
\begin{aligned}
& P_{s}\left(d_{s}, z\right)=\alpha k_{s} d_{s}+(1-\alpha) k_{s} d_{y s} z \\
& \dot{z}=\frac{1}{d_{y s}}\left(\dot{d}_{s}-\gamma\left|\dot{d}_{s}\right| z|z|^{n-1}-\beta \dot{d}_{s}|z|^{n}\right)
\end{aligned}
$$

The simulation result shown in figure 4 shows that even the nonlinear effect exists in the specimen, MPC is still robust enough to achieve good control performance and improve the time delay issue. 


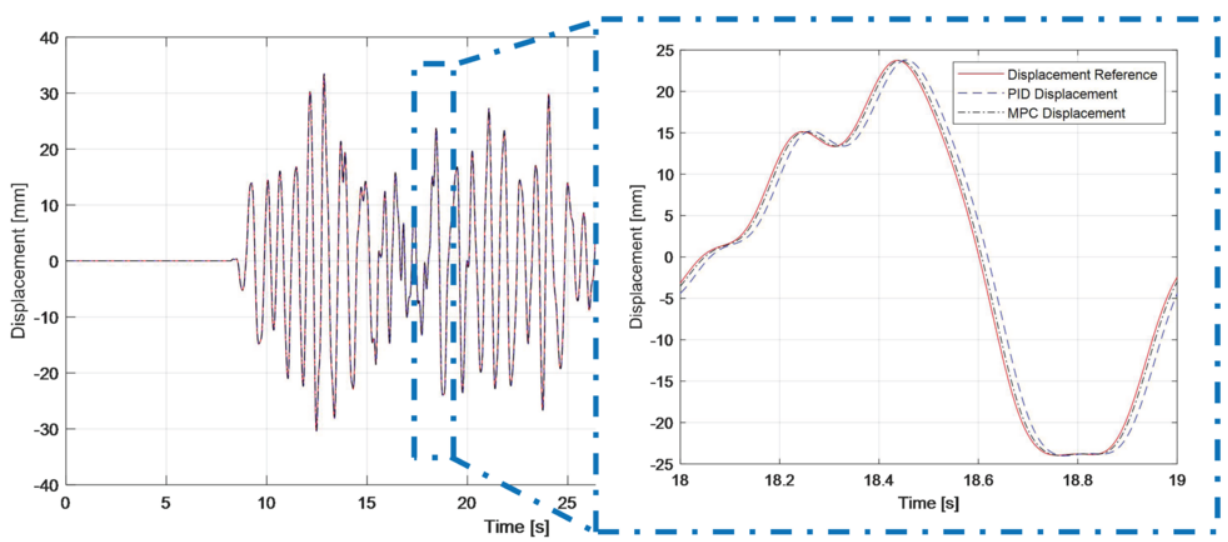

(a) Time history comparison

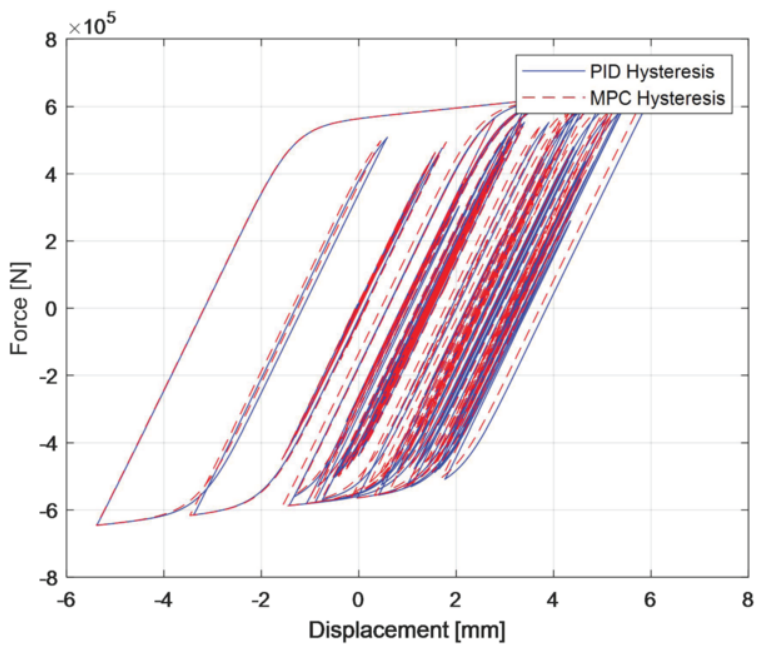

(b) Specimen hysteresis

Figure 4. Nonlinear specimen simulation result

\section{Conclusion}

In this study, MPC is introduced in time delay compensation indued by the nonlinear dynamics of the shake table and the CSI effect. A Kalman filter is used to provide MPC with future plant output, in order to derive the optimal control sequence. In this paper, the dynamic model including the shake table control system and a SDOF specimen was established, and its parameters were identified using grey-box identification method. Then, the MPC controller based on the control model was addressed, followed by two numerical cases, with linear and nonlinear specimens. Results from two cases illustrate that both the tracking performance and time delay are improved and validate that the proposed MPC has a receiving effectiveness and robustness. Further work will focus on conducting a real RTHS test using the proposed MPC strategy. 


\section{Acknowledgments}

The authors are grateful for the financial support from the National Natural Science Foundation of China (Project No. 51878674, 51878563). Any opinions, findings, and conclusions or recommendations expressed in this study are those of the authors.

\section{References}

[1] Horiuchi T, Inoue M, Konno T, Namita Y. Real-time hybrid experimental system with actuator delay compensation and its application to a piping system with energy absorber. Earthquake Engineering \& Structural Dynamics. 1999;28(10):1121-41.

[2] Dyke SJ, Spencer BF, Quast P, Sain MK. Role of Control-Structure Interaction in Protective System Design. Journal of Engineering Mechanics. 1995 Feb 1;121(2):322-38.

[3] Chae Y, Kazemi Bidokhti K, Ricles J. Adaptive time series compensator for delay compensation of servo-hydraulic actuator systems for RTHS. Earthquake Engineering \& Structural Dynamics. 2013 Sep $1 ; 42$.

[4] Najafi A, Spencer BF. Adaptive model reference control method for real-time hybrid simulation. Mechanical Systems and Signal Processing. 2019 Oct 1;132:183-93.

[5] Ryu KP, Reinhorn AM. Real-time control of shake tables for nonlinear hysteretic systems. Structural Control and Health Monitoring. 2017;24(2):e1871.

[6] Haukaas T, Der Kiureghian A. Finite element reliability and sensitivity methods for performance-based earthquake engineering. 2003. 